\title{
Effects of serial position and set size in auditory recognition memory*
}

\author{
ARTHUR WINGFIELD \\ Brandeis University, Waltham, Massachusetts 02154
}

\begin{abstract}
Auditorially presented lists of from one to six digits were memorized by Ss with instructions to scan the memory set to determine the presence or absence of a single auditorially presented probe digit. Results showed cooccurrence of parallel linear increases in decision time with list length for positive and negative responses and effects of serial position of probes in the memory lists. A comparison with two previous studies using visual presentation of materials suggests faster encoding of auditorially presented probes but similarity in processing time in memory once probes have been encoded.
\end{abstract}

Studies of rapid processing of temporarily held information soon after receipt, and prior to much memory decay, have received considerable attention in recent years. In the experimental paradigm considered here, $\mathrm{S}$ was given a list of digits for memory, followed at a short interval by a single "probe" digit. S's task was to indicate whether or not the probe item was present in the memorized list. A frequent finding was that of a linear increase in decision reaction time (RT) with the number of items in the memory set, similarity in slopes of RT on set size for "yes" and "no" responses, and an absence of effects on RT of the serial or temporal position of the probe item in the memorized list.

Two general classes of models have been introduced to account for these and related findings. The first, a serial exhaustive scan model, proposes an item-by-item comparison of the probe against each item in memory with all items sampled prior to response (Sternberg, 1966). Serial processing is implied by the systematic increase in RT with each item added to the memory set, while the postulate of exhaustive sampling is derived from comparable rates of increase for the "yes" responses and the necessarily exhaustive "no" responses. Equality of processing time for each item in the serial comparison stage has been a usual simplifying assumption (Sternberg, 1969). The serial character of the search process has received support even in such cases as the use of multiple probes where the generality of exhaustive scanning has been questioned (Wingfield \& Bolt, 1970). The second class of theoretical models recently applied to this paradigm is the possibility of parallel processing, in which sampling of all items in the memory set are presumed to begin and continue simultaneously, with variance in RT reflecting differences in completion time for the items either within a given memorized list or between lists of different lengths. While more complex, such models can

\footnotetext{
*This study was supported in part by PHS Grant NS 09767-01 from the National Institutes of Health.
}

also be shown, at least in principle, to account for much of the memory search data obtained (Townsend, 1971; Murdock, 1971).

Effects on RT of a probe's serial position in the memory set, a finding counter to the notion of an exhaustive serial scan, has been found with invariant list lengths (Corballis, 1967; Morin, DeRosa \& Stultz, 1967). Such findings might well reflect alternative search or rehearsal strategies when fixed or varying memory set sizes are encountered by Ss. The recent findings of Burrows and Okada (1971) of a cooccurrence of parallel slopes for "yes" and "no" responses and significant serial position effects, however, is intriguing. The present communication reports similar results for auditory presentation of both memory lists and probes, and also compares search rates obtained with those of previous

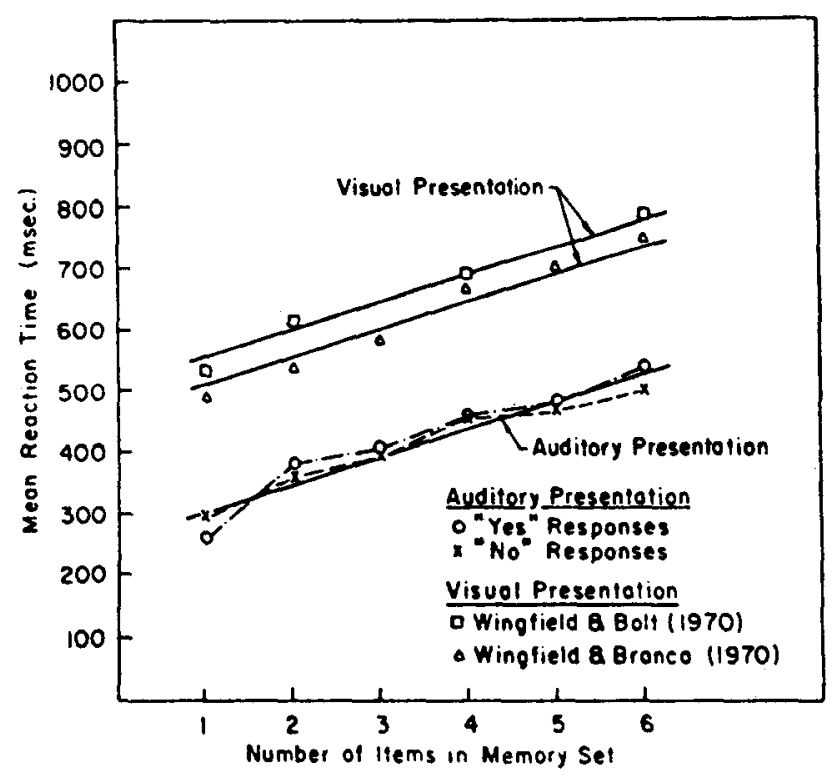

Fig. 1. Mean RT to "yes" and "no" responses as a function of memory set size for auditory presentation of lists and probes. shown with parallel functions fitted to similar data obtained with visual presentations. 


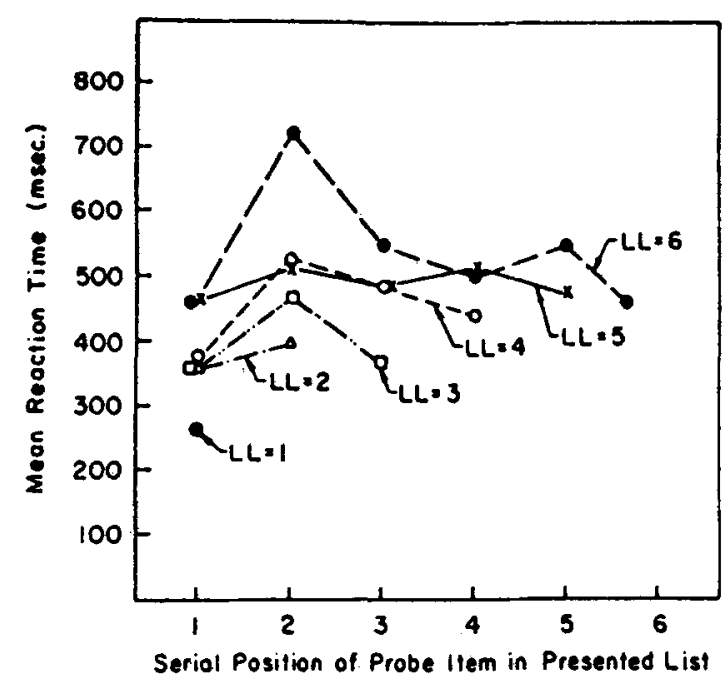

Fig. 2. Mean RT as a function of serial position of probe item in memory lists for auditory presentation of lists and probes.

studies employing the more usual visual presentation of memory and probe stimuli.

\section{METHOD}

The Ss heard recorded lists of from one to six spoken digits at a rate of one digit every $500 \mathrm{msec}$, followed after a $1,500-\mathrm{msec}$ delay by a single spoken digit serving as the "probe" item. Sibnuli were heard through high-quality stereo earphones, with digits of the memory set presented to one ear and the probe item to the other. Occurrence of the probe activated a Hunter 1520 reaction timer through operation of a voice-operated relay monitoring the recorder channel bearing the recorded probe. The RTs were terminated when $S$ pressed one of two levers to indicate whether the probe had or had not been in the memorized list. The Ss, 12 university undergraduates, were instructed to respond as rapidly as possible without making careless errors.

Each S heard 12 lists of each of the six lengths used, with probe digits present in half of the lists heard. When the probe was present, its serial position in the memory set was varied systematically. All lists of a given length were presented in a block of trials, with each block preceded by six practice lists of that length. Polarity of response levers, as well as order of presentation of list length blocks, was balanced across Ss.

\section{RESULTS AND DISCUSSION}

Figure 1 shows mean RT for correct "yes" and "no" responses plotted as a function of the number of digits in the memorized lists. The overall error rate was $2.9 \%$, approximately equally distributed between false positives and false negatives, with a slight trend toward an increase in errors with size of the memory set (from $1.4 \%$ for one-digit to $4.9 \%$ for six-digit lists). The RTs to both "yes" and "no" responses increased linearly with the size of the memory set, and their slopes are similar (49.9 msec per digit for "yes" and $40.8 \mathrm{msec}$ per digit for "no" responses). The effect of list length on RT was significant $[F(5,55)=23.50, p<.001]$. The effect of response type ("yes" vs "no") was not significant
$[F(1,11)<1]$, nor was there a significant List Length by Response Type interaction.

These results agree with those obtained with visually' presented stimuli, both in respect to the parallel slopes of "yes" and "no" responses, with their implication of serial exhaustive search, and to absolute value of the slope constants themselves. This latter point is illustrated in Fig. 1, which also shows data taken from comparable portions of two previous studies using visual presentations (Wingfield \& Branca, 1970; Wingfield \& Bolt, 1970). In both cases, digit lists were visually displayed for durations representing $1 \mathrm{sec}$ per digit in the list, followed after a $1-\mathrm{sec}$ interval by a visually presented probe. Error rates were $3.6 \%$ and $6.6 \%$ and did not increase systematically with list length. Since response type was not a significant factor in either study, points shown in Fig. 1 are mean values for positive and negative responses at each list length. While mean slopes for these experiments do vary $(49.1 \mathrm{msec}$ per digit for Wingfield and Bolt vs $57.1 \mathrm{msec}$ for Wingfield and Branca), the larger differences are in heights of the functions along the ordinate. This is emphasized by the fitting of parallel functions derived from the slope of the auditory experiment to all three sets of data. The fit is visually good and was confirmed by analysis of variance.

The fitting of parallel functions implies similarity in processing rate in memory once the probe has been encoded for internal companison. The difference between $y$-intercepts (encoding time of the probe) for the two visual experiments is relatively small and probably attributable to differences in display characteristics in these two studies. The much larger difference is in the $y$-intercept for auditory presentation, suggesting more rapid encoding of the auditory as opposed to the visual probes, for a more compatible matching in a presumably acoustic memory store.

Unlike the two visual studies cited, the present auditory experiment showed effects on RT of the serial position of the probe in the memorized list. These data are plotted in Fig. 2 for each of the six list lengths (LLs) used. Trends toward both primacy and recency effects can be seen, although they are somewhat inconsistent, reaching significance in analysis of variance for lists of three $(p<.001)$, four $(p<.05)$, and six $(p<.001)$ items, but not for list lengths of two or five.

The presence in these auditory data of linear, parallel functions for positive and negative RTs with set size, along with effects of serial position, can be added to those data of Burrows and Okada (1971), who report similar paradoxical findings for visual presentations of lists and probes. The existence of serial position effects are counter to the simplest notion of exhaustive search, while the similarity in slope constants would appear to argue against a serial self-terminating search where "yes" responses would, on average, increase at half the rate of "no" responses. It is certainly the case that such findings cannot in themselves distinguish between parallel and 
serial processing in memory. Rather, they do encourage a continued consideration of the importance of possible differences in comparison time for items within a list and, by implication, raise the possibility of differences in mean processing time for items across lists of various lengths. Whether such differences might be attributable to differential trace strength of items in memory (Baddeley \& Ecob, 1970; Wingfield \& Byrnes, 1972), or perhaps to a shift of criteria as the comparison process continues, it seems increasingly likely that the simple elegance of an unmodified serial exhaustive scan model must be sacrificed for a more complete account of retrieval from working memory.

\section{REFERENCES}

Baddeley, A. D., \& Ecob, J. R. Reaction time and short-term memory: A trace strength alternative to the high-speed exhaustive scanning hypothesis. Technical Report No. 13, Center for Human Information Processing, University of California at San Diego, 1970.

Burrows, D., \& Okada, R. Serial position effects in high-speed memory search. Perception \& Psychophysics, 1971, 10, 305-308.

Corballis, M. Serial order in recognition and recall. Journal of Experimental Psychology, 1967, 74, 99-105.

Morin, R. E., DeRosa, D. V., \& Stultz, V. Recognition memory and reaction time. Acta Psychologica, 1967, 27, 298-305.

Murdock, B. B. A parallel-processing model for scanning. Perception \& Psychophysics, 1971, 10, 289-291.

Sternberg, S. High-speed scanning in human memory. Science, $1966,153,652-654$.

Sternberg, S. Memory scanning: Mental processes revealed by reaction-time experiments. American Scientist, 1969, 57, 421-457.

Townsend, J. T. A note on the identifiability of parallel and serial processes. Perception \& Psychophysics, 1971, 10, $161-163$.

Wingfield, A., \& Branca, A. A. Strategy in high-speed memory search. Journal of Experimental Psychology, 1970, 83, 63-67.

Wingfield, A., \& Bolt, R. A. Memory search for multiple targets. Journal of Experimental Psychology, 1970, 85, 45-50.

Wingfield, A., \& Byrnes, D. L. Decay of information in short-term memory. Science, 1972, 176, 690-692.

(Received for publication July 10, 1972; accepted September 12, 1972.) 